\title{
On the maximum degree of path-pairable planar graphs
}

\author{
António Girão \\ Department of Pure Mathematics and Mathematical Statistics \\ University of Cambridge \\ Cambridge, U.K. \\ a.girao@dpmms.cam.ac.uk \\ Gábor Mészáros Kamil Popielarz Richard Snyder
Department of Mathematical Sciences
The University of Memphis
Memphis, Tennessee, U.S.A.
gmeszaros@purestorage.com, \{kamil.popielarz,rjsnyder23\}@gmail.com
}

Submitted: Sep 6, 2017; Accepted: Apr 2, 2019; Published: May 3, 2019

(c) The authors. Released under the CC BY license (International 4.0).

\begin{abstract}
A graph is path-pairable if for any pairing of its vertices there exist edge-disjoint paths joining the vertices in each pair. We investigate the behaviour of the maximum degree in path-pairable planar graphs. We show that any $n$-vertex path-pairable planar graph must contain a vertex of degree linear in $n$. Our result generalizes to graphs embeddable on a surface of finite genus.
\end{abstract}

Mathematics Subject Classifications: 05C07, 05C10, 05C35, 05C40

\section{Introduction}

We are interested in path-pairability, a graph theoretical notion that emerged from a practical networking problem. This notion was introduced by Csaba, Faudree, Gyárfás, Lehel, and Schelp [3], and further studied by Faudree, Gyárfás, and Lehel [4, 5, 6], and by Kubicka, Kubicki and Lehel [14]. Given a fixed integer $k$ and a simple undirected graph $G$ on at least $2 k$ vertices, we say that $G$ is $k$-path-pairable if, for any pair of disjoint sets of distinct vertices $\left\{x_{1}, \ldots, x_{k}\right\}$ and $\left\{y_{1}, \ldots, y_{k}\right\}$ of $G$, there exist $k$ edge-disjoint paths $P_{1}, P_{2}, \ldots, P_{k}$, such that $P_{i}$ is a path from $x_{i}$ to $y_{i}, 1 \leqslant i \leqslant k$. The problems of finding $k$ edge(vertex)-disjoint paths routing some prescribed pairs of vertices in a graph is a well-known problem in algorithmic graph theory and combinatorial optimization (see the 
surveys[7, 8, 20]). Recently, for a fixed integer $k$, Kawarabayashi, Kobayashi and Reed [13] constructed a $O\left(n^{2}\right)$ time algorithm which for any graph $G$ on $n$ vertices either finds such $k$ vertex-disjoint paths or concludes no such paths exist. As a corollary they obtained a $O\left(n^{2}\right)$ time algorithm for the edge-disjoint path problem. This improved upon the seminal work of Robertson and Seymour [18], which initially gave a $O\left(n^{3}\right)$ time algorithm for the vertex-disjoint path problem. Note that the problem of finding edge(vertex)-disjoint paths between an unbounded number of prescribed pairs of vertices is known to be NP-complete, even when restricted to planar graphs [17].

The concept of $k$-path-pairability is closely related to the well-studied notions of $k$ linkedness and $k$-weak-linkedness. A graph is said to be $k$-(weakly)linked if for any choice $\left\{s_{1}, \ldots, s_{k}, t_{1}, \ldots, t_{k}\right\}$ of $2 k$ vertices (not necessarily distinct) there are vertex(edge) internally disjoint paths $P_{1}, \ldots, P_{k}$ with $P_{i}$ joining $s_{i}$ to $t_{i}, 1 \leqslant i \leqslant k$. While any $k$ (weakly)linked graph is $(2 k-1)$-vertex connected ( $k$-edge connected), the same need not hold for $k$-path-pairable graphs. Observe that the stars $S_{2 k}(k \geqslant 1)$ are $k$-path-pairable and yet have very low edge density and edge connectivity. On the other hand, a result of Bollobás and Thomason [2] shows that if $G$ is a $2 k$-connected graph with average degree at least $22 k$ then $G$ is $k$-linked. This was later improved by Thomas and Wollan [19] who showed that a $2 k$-connected graph with average degree at least $10 k$ is necessarily $k$-linked. In the context of weakly-linked graphs, a theorem of Hirata, Kubota and Saito [11] states that a $(2 k+1)$-edge connected graph is $(k+2)$-weakly-linked for $k \geqslant 2$. A few years later, Huck [12] showed that any $(k+2)$-edge-connected graph is $k$-weakly-linked.

A $k$-path-pairable graph on $2 k$ vertices is simply said to be path-pairable. Some of the most central questions in the study of path-pairable graphs concern determining the behaviour of their maximum degree. It is fairly easy to construct path-pairable graphs on $n$ vertices ( $n$ even) with maximum degree linear in $n$. For example, complete graphs $K_{2 n}$ and complete bipartite graphs $K_{m, n}$ are path-pairable for all choices of $m, n \in \mathbb{N}$ with $m+n$ even, $m \neq 2, n \neq 2$.

It is slightly more challenging to construct an infinite family of path-pairable graphs where the maximum degree grows sublinearly. We shall now describe such a family. Let $K_{t}$ be the complete graph on $t$ vertices and let $K_{t}^{q}$ be constructed from $K_{t}$ by attaching $q-1$ leaves to each of the original vertices of $K_{t}$. This family was introduced by Csaba, Faudree, Gyárfás, and Lehel [3], who also proved that $K_{t}^{q}$ is path-pairable as long as $t \cdot q$ is even and $q \leqslant\left\lfloor\frac{t}{3+2 \sqrt{2}}\right\rfloor$. The bound on $q$ has been recently improved to $\approx \frac{1}{3} t$ [9]. Observe that $n=\left|V\left(K_{t}^{q}\right)\right|=t \cdot q$ and $\Delta\left(K_{t}^{q}\right)=t+q-2=O(\sqrt{n})$ when $q=\Omega(t)$. Additional path-pairable constructions with maximum degree $c \sqrt{n}$ can be found in [14] and [16].

The following result due to Faudree [5] shows that the maximum degree of a pathpairable graph has to grow with the order of the graph.

Theorem 1. If $G$ is path-pairable on $n$ vertices with maximum degree $\Delta$, then $n \leqslant 2 \Delta^{\Delta}$.

Letting $\Delta_{\min }(n):=\min \{\Delta(G): G$ is a path-pairable graph on $n$ vertices $\}$, this result implies that

$$
\Delta_{\min }(n) \geqslant c_{1} \frac{\log n}{\log \log n}
$$


for some constant $c_{1}$. To date, the best known upper bound on $\Delta_{\min }(n)$ is due to Győri, Mezei, and Mészáros, exhibiting a path-pairable graph with maximum degree $\Delta \approx 5.5 \cdot \log n[10]$. In summary, we have the following general asymptotic bounds on $\Delta_{\min }(n)$ :

$$
c_{1} \frac{\log n}{\log \log n} \leqslant \Delta_{\min }(n) \leqslant c_{2} \log n .
$$

Recall that the star $K_{1, n-1}$ is path-pairable. This is simply due to the presence of a vertex of large degree. Are there properties we may impose on a general path-pairable graph to force a vertex of large degree, say, linear in $n$ ? Along these lines, Faudree, Gyárfás and Lehel [6] proved that an $n$-vertex path-pairable graph with maximum degree at most $n-2$ must have at least $3 n / 2-\log n-c$ edges, for some absolute constant $c$. Instead of simply imposing a condition on the number of edges, we wished to determine whether or not a structural property like planarity would be enough to force a vertex of linear degree in a path-pairable graph. To formulate this precisely, let us define $\Delta_{\text {min }}^{p}(n)$ to be

$$
\min \{\Delta(G): G \text { is a path-pairable planar graph on } n \text { vertices }\} .
$$

Our problem, then, is to determine whether or not $\Delta_{\min }^{p}(n)=\Theta(n)$. We first note that a simple application of the Planar Separator Theorem of Lipton and Tarjan [15] shows that every path-pairable planar graph on $n$ vertices must contain a vertex of degree at least $c \sqrt{n}$. Indeed, if $G$ is such a graph, then the Separator Theorem allows us to partition $V(G)$ into three sets $S, A, B$, where $|S|=O(\sqrt{n}),|A| \leqslant|B| \leqslant 2 n / 3$, and there are no edges between $A$ and $B$. Now, while path-pairable graphs $G$ need not be highly connected or edge connected, they must satisfy certain connectivity-like conditions. More precisely, they must satisfy the cut-condition: for every subset $X \subset V(G)$ of size at most $n / 2$, there are at least $|X|$ edges between $X$ and $V(G) \backslash X$. Note that the cut-condition is not sufficient to guarantee path-pairability; see [16] for additional details. Accordingly, since $n / 4<|A|<n / 2$ and there are no edges between $A$ and $B$, the cut-condition implies that there are at least $|A|$ edges between $A$ and $S$. We therefore obtain a vertex in $S$ of degree $\Omega(\sqrt{n})$.

Our main theorem, which we state below, shows that we can do much better than this. Namely, every path-pairable planar graph must have a vertex of linear degree.

Theorem 2. There exists $c \geqslant 10^{-10^{10}}$ such that if $G$ is a path-pairable planar graph on $n$ vertices then $\Delta(G) \geqslant c n$.

We have not made an attempt to optimize the constant $c$ obtained in the proof. The value we give is surely far from the truth.

In the other direction, there are easy examples of path-pairable planar graphs with very large maximum degree; for example, consider the star $K_{1, n-1}$. Our second result finds an infinite family of path-pairable planar graphs with smaller (but of course still linear) degree.

Theorem 3. There exist path-pairable planar graphs $G$ on $n$ vertices with $\Delta(G)=\frac{2}{3} n$. 
Combining Theorems 2 and 3, we have that

$$
10^{-10^{10}} n \leqslant \Delta_{\min }^{p}(n) \leqslant \frac{2}{3} n
$$

However, there is currently a significant gap between the constants in the upper and lower bounds. Closing this gap and finding the truth is an interesting open problem.

We remark that using the same techniques it is possible to generalize these results to path-pairable graphs which can be embedded on a surface of a finite genus.

\subsection{Organization}

The remainder of the paper is organized as follows. In the next short section, we shall describe our construction establishing Theorem 3. The third section of this paper contains a proof of our main theorem, Theorem 2. This proof relies on three preparatory lemmas and on some common facts concerning planar graphs. In particular, we use heavily the fact that any subset $X$ of the vertices of a planar graph induces less than $3|X|$ edges, and any bipartite planar graph on $n$ vertices has less than $2 n$ edges. Finally, we close with some remarks and open problems.

\subsection{Notation}

Our notation is standard. Thus, for a graph $G$ and two subsets $X, Y \subset V(G)$ we say that a path in $G$ is an $X-Y$ path if it begins in $X$ and ends in $Y$. If $X=\{x\}$ and $Y=\{y\}$ are singletons, we shall simply say that the path is an $x-y$ path. For subsets $X, Y \subset V(G), e(X, Y)$ is the number of edges with one endpoint in $X$ and the other in $Y$. As usual, $G[X]$ denotes the graph induced in $G$ with vertex set $X$.

\section{The Construction}

Our aim in this section is to prove Theorem 3, which we restate here for convenience.

Theorem 3. There exist path-pairable planar graphs $G$ on $n$ vertices with $\Delta(G)=\frac{2}{3} n$.

Proof. Let $G$ be a graph on $n=6 k$ vertices with vertex set $V(G)=A \cup B \cup C \cup$ $\left\{x_{A B}, x_{B C}, x_{C A}\right\}$ where $|A|=|B|=|C|=2 k-1$, and $x_{A B}, x_{B C}, x_{C A}$ denote three additional vertices forming a triangle such that $x_{A B}, x_{B C}, x_{C A}$ are joined to every vertex in $A \cup B, B \cup C$, and $C \cup A$, respectively, and $A, B, C$ are independent sets. This graph is clearly planar. Let $\mathcal{P}$ be a pairing of the vertices and let $\{u, v\} \in \mathcal{P}$. We describe how to join $u$ and $v$ by a path in all possible cases.

1. If there is an edge between $u$ and $v$, join them by this edge.

2. If $u \in\left\{x_{A B}, x_{B C}, x_{C A}\right\}$ and $v \in A \cup B \cup C$ such that there is no edge between them, join them by the path $u w v$ where the edge $u w$ is consistent with the cyclic ordering $x_{A B}, x_{B C}, x_{C A}$. For example, if $u=x_{A B}$ and $v \in C$, we join $u$ and $v$ by the path $u x_{B C} v$. The remaining cases can be dealt using the same pattern. 
3. If $u, v \in A \cup B \cup C$ and they are in the same class, join them by the path $u w v$ where $w$ is an arbitrary common neighbour (out of the two available).

4. If $u, v \in A \cup B \cup C$ and they are in different classes, join them by the path $u w v$ where $w$ is the unique common neighbour.

It is straightforward to check that the above instructions find edge-disjoint paths joining terminals, regardless of the choice of $\mathcal{P}$.

\section{The Proof of Theorem 2}

The aim of this section is to prove our main theorem, Theorem 2. Our proof is based on three preparatory lemmas. First, we shall introduce some terminology. Let $G$ be a multigraph. We say that two multiedges $e, f$ of $G$ are at distance $d$ if the shortest path in $G$ joining an endpoint of $e$ and an endpoint of $f$ has length $d$. If two multiedges are at distance 0 , we shall simply say they are incident. Further, we shall refer to a matching of size $k$ as a $k$-matching. We say that a $k$-matching is good if every pair of edges in the matching is at distance exactly 1 . Notice that contracting all the edges of a good $k$-matching results in the complete graph $K_{k}$ (with potential multiple edges and loops).

If we consider the complete graph whose vertices are the multiedges of some graph, and 3-colour its edges according to certain notions of 'density', then by Ramsey's theorem one of the colours must contain a 'dense' subgraph. Our first lemma is a Ramsey-type result in a similar vein (although we do not need to appeal to Ramsey's theorem itself). More precisely, our lemma says that in any multigraph with enough multiedges, either some multiedges cluster together, or many pairs of multiedges are far apart, or one can find a good $k$-matching. We first need the following inequality.

Fact 4. If $k \geqslant 2$ then $2^{-k}\left(\frac{1+2^{-k-1}}{\left(1-2^{-k}\right)^{2}}\right) \leqslant 2^{-k+1}$.

The above inequality is easily seen to be equivalent to $\left(2^{-k+2}-1\right)\left(2^{-k-1}-1\right) \geqslant 0$.

Lemma 5. Let $k$ be a natural number and $\varepsilon_{1}, \varepsilon_{2}$ be positive reals such that $\varepsilon_{1}+\varepsilon_{2} \leqslant 2^{-k}$. Then, for sufficiently large $M=M(k)$, if $G$ is a multigraph on $M$ multiedges, then at least one of the following conditions is satisfied.

1. There is a multiedge in $G$ which is incident with at least $\varepsilon_{1} M$ multiedges;

2. There are at least $\varepsilon_{2}\left(\begin{array}{c}M \\ 2\end{array}\right)$ pairs of multiedges which are at distance greater than 1 ;

3. $G$ contains a good k-matching.

Proof. We shall use induction on $k$. The base case when $k=1$ is trivial - Condition 3 is always satisfied. Assume then that $k \geqslant 2$ and the lemma is true for $k-1$.

Suppose every multiedge is incident with at most $\varepsilon_{1} M$ multiedges and at most $\varepsilon_{2}\left(\begin{array}{c}M \\ 2\end{array}\right)$ pairs of multiedges are at distance greater than 1 . We shall show that $G$ contains a good $k$-matching. By an averaging argument there is a multiedge $e$ which is at distance at 
most 1 from at least $\left(1-\varepsilon_{2}\right) M-1$ multiedges. Let $E^{\prime}$ be the set of those multiedges which are at distance exactly 1 from $e$. It follows from our assumptions that $M^{\prime}=$ $\left|E^{\prime}\right| \geqslant\left(1-\varepsilon_{1}-\varepsilon_{2}\right) M-1 \geqslant\left(1-2^{-k}\right) M-1$. Let $G^{\prime}$ be the multigraph spanned by $E^{\prime}$. By assumption, at most $\varepsilon_{2}\left(\begin{array}{c}M \\ 2\end{array}\right)$ of the multiedges in $G^{\prime}$ are at distance greater than 1 . Therefore, since $M \leqslant \frac{M^{\prime}+1}{1-2^{-k}}$, for large enough $M$ (and hence large enough $M^{\prime}$ ) we have that at most

$$
\begin{aligned}
\varepsilon_{2}\left(\begin{array}{c}
M \\
2
\end{array}\right) \leqslant \varepsilon_{2}\left(\begin{array}{c}
\frac{M^{\prime}+1}{1-2^{-k}} \\
2
\end{array}\right) & =\frac{\varepsilon_{2}}{\left(1-2^{-k}\right)^{2}}\left(1+\frac{1}{M^{\prime}}\right)\left(1+\frac{1+2^{-k}}{M^{\prime}-1}\right)\left(\begin{array}{c}
M^{\prime} \\
2
\end{array}\right) \\
& \leqslant \frac{\varepsilon_{2}\left(1+2^{-k-1}\right)}{\left(1-2^{-k}\right)^{2}}\left(\begin{array}{c}
M^{\prime} \\
2
\end{array}\right),
\end{aligned}
$$

pairs of multiedges in $G^{\prime}$ are at distance greater than 1. Also, for $M^{\prime}$ large enough, each multiedge in $G^{\prime}$ is incident with at most $\varepsilon_{1} M \leqslant \varepsilon_{1} \frac{M^{\prime}+1}{1-2^{-k}}=\frac{\varepsilon_{1}}{1-2^{-k}}\left(1+\frac{1}{M^{\prime}}\right) M^{\prime} \leqslant$ $\frac{\varepsilon_{1}\left(1+2^{-k-1}\right)}{1-2^{-k}} M^{\prime}$ multiedges. Note that for $k \geqslant 2$ one has

$$
\begin{aligned}
\varepsilon_{1} \frac{1+2^{-k-1}}{1-2^{-k}}+\varepsilon_{2} \frac{1+2^{-k-1}}{\left(1-2^{-k}\right)^{2}} & \leqslant \varepsilon_{1} \frac{1+2^{-k-1}}{\left(1-2^{-k}\right)^{2}}+\varepsilon_{2} \frac{1+2^{-k-1}}{\left(1-2^{-k}\right)^{2}} \\
& \leqslant 2^{-k} \frac{1+2^{-k-1}}{\left(1-2^{-k}\right)^{2}} \leqslant 2^{-(k-1)},
\end{aligned}
$$

where the last inequality is precisely Fact 4. Therefore, by the induction hypothesis, $G^{\prime}$ contains a good $(k-1)$-matching. But since $e$ is at distance 1 from any multiedge in $G^{\prime}$, we also have a good $k$-matching in $G$.

Since we shall be operating with planar graphs, we single out the following corollary.

Corollary 6. Let $M$ be a sufficiently large integer and let $\varepsilon_{1}, \varepsilon_{2}$ be positive reals such that $\varepsilon_{1}+\varepsilon_{2} \leqslant \frac{1}{32}$. If $G$ is a planar multigraph with $M$ multiedges then either $G$ has a multiedge which is incident with at least $\varepsilon_{1} M$ multiedges or there are at least $\varepsilon_{2}\left(\begin{array}{c}M \\ 2\end{array}\right)$ pairs of multiedges at distance greater than 1.

Proof. If $G$ contained a good 5-matching then it would contain a $K_{5}$ minor.

We say that that a pairing of vertices of a graph contributes to $k$ edges if any system of edge-disjoint paths joining the vertices of each pair uses at least $k$ edges. One strategy in the proof of our main theorem is to consider a suitable bipartition of our path-pairable planar graph, and to exploit the fact that any bipartite planar graph on $n$ vertices has at most $2 n-4$ edges. To exploit this last property we shall need ways of finding pairings of the vertices which contribute to 'many' edges to the bipartition. This is formalized in the following lemma.

Lemma 7. Let $D$ be an integer and $0<\varepsilon \leqslant 1 / 2$. Then there exists $c>0$ such that the following is true. Suppose $G$ is a path-pairable planar graph on $n$ vertices with $\Delta=$ $\Delta(G) \leqslant c n$. Let $A, U \subset V(G)$ be given with $U \subset A$ such that every vertex in $A$ has degree at most $D,|A| \geqslant(1-\varepsilon) n$ and $|U| \geqslant \varepsilon n$. Let $B=V(G) \backslash A$. Then there is a pairing of the vertices in $U$ which contributes to at least $2|U|-16 \varepsilon n$ edges between $A$ and $B$. 
Proof. We say that a path in $G$ is weak if it begins and ends in $A$, uses no edges inside $B$, and uses at most 2 edges between $A$ and $B$. Now, let $C:=\left\lceil 4 \varepsilon^{-1}\right\rceil$ and note that since $\varepsilon \leqslant 1 / 2$ we have that $\frac{3}{C-2} \leqslant \varepsilon$. For every $x \in U$, let $X(x)=\left\{u \in U: \operatorname{dist}_{G}(u, x) \leqslant C\right\}$ and $Y_{x}=\{u \in U: \exists$ a weak $x-u$ path in $G\}$. Finally, consider the set $U_{x}=X(x) \cap Y_{x}$. We claim that $U_{x}$ is small for every $x \in U$. So fix $x \in U$ and consider the vertices reachable from $x$ at distance at most $C$ using only weak paths. Such a path can either stay entirely in $A$, or use precisely two edges between $A$ and $B$. In the first case, since vertices in $A$ have degree at most $D$, the number of such vertices in $U_{x}$ is at most $D^{C}$. To bound the number of vertices reachable from paths of the second type, note that such a path can first move around inside $A$, then leave $A$ and use precisely two edges between $A$ and $B$ (and no edges inside $B$ ), then finally move around inside $A$ again. Recalling that the maximum degree of $G$ is $\Delta$ and the maximum degree of vertices in $A$ is $D$, we obtain the bound $D^{C} \cdot D \Delta \cdot D^{C}$. It follows that

$$
\left|U_{x}\right| \leqslant D^{C}+D^{C} D \Delta D^{C}=D^{C}\left(1+D^{C+1} \Delta\right) \text {. }
$$

Choose $c=c(D, \varepsilon)=\frac{\varepsilon}{4 D^{2 C+1}}$ so that $\Delta \leqslant c n$. Then $\left|U_{x}\right| \leqslant D^{C}\left(1+D^{C+1} \Delta\right) \leqslant$ $\left(D^{C}+D^{2 C+1}\right) \Delta$, and using the upper bound on $\Delta$, we find that

$$
\left|U_{x}\right| \leqslant \varepsilon n / 2 .
$$

Let us define an auxiliary graph $G_{U}$ with vertex set $U$ where we join two vertices $x, y$ provided $y \notin U_{x}$ (equivalently, $x \notin U_{y}$ ). It is easy to see that $G_{U}$ has a perfect matching (or 'almost' perfect, if $|U|$ is odd; this makes no difference for us). Indeed, the degree of every vertex in $G_{U}$ is at least $|U|-\frac{\varepsilon}{2} n \geqslant|U| / 2$, and therefore $G_{U}$ has a Hamilton cycle. Fix a perfect matching $\mathcal{M}$ in $G_{U}$ according to this Hamilton cycle and fix a pairing $\mathcal{P}$ of the vertices of $G$ where each edge of $\mathcal{M}$ forms a pair. Finally, since $G$ is path-pairable, choose a collection of edge-disjoint paths $\mathcal{R}$ that connect the vertices of each pair in $\mathcal{P}$. Observe that any path from $\mathcal{R}$ must use an even number of edges between $A$ and $B$. We single out two types of edges $e=x y$ in $\mathcal{M}$ with respect to $\mathcal{R}$ : either the $x-y$ path in $\mathcal{R}$ is weak but $\operatorname{dist}(x, y)>C$, or this $x-y$ path uses at least 4 edges between $A$ and $B$. Let $\mathcal{M}=\mathcal{E}_{0} \cup \mathcal{E}_{1} \cup \mathcal{E}_{2}$, where $\mathcal{E}_{0}$ denotes the edges satisfying the former condition, $\mathcal{E}_{1}$ the latter, and $\mathcal{E}_{2}$ denotes the remaining edges. We claim that most edges are in $\mathcal{E}_{1}$. Indeed, observe that if $e=x y \in \mathcal{E}_{2}$, then the $x-y$ path must use edges from $B$. By planarity we have $e(B)<3|B|$, and therefore $\left|\mathcal{E}_{2}\right|<3 \varepsilon n$. Using planarity again we have that $e(A)<3|A|$. On the other hand, for each edge in $\mathcal{E}_{0}$ its path in $\mathcal{R}$ uses more than $C$ edges, at most 2 of which are in the cut $\{A, B\}$, and none of which belong to $B$. Accordingly, since these paths are edge-disjoint, we have that $e(A) \geqslant(C-2)\left|\mathcal{E}_{0}\right|$ and so

$$
\left|\mathcal{E}_{0}\right|<\frac{3}{C-2}|A| \leqslant \varepsilon|A|
$$

Therefore, $\left|\mathcal{E}_{1}\right| \geqslant \frac{1}{2}|U|-\varepsilon|A|-3 \varepsilon n \geqslant \frac{1}{2}|U|-4 \varepsilon n$. It follows that since every path in $\mathcal{R}$ connecting an edge in $\mathcal{E}_{1}$ contributes at least 4 edges between $A$ and $B$, and these paths must be edge-disjoint, we have

$$
e(A, B)>2|U|-16 \varepsilon n .
$$


This completes the proof of Lemma 7.

Our final lemma allows us to quantify more precisely the degree distribution in any bipartite planar graph.

Lemma 8. Let $G$ be a bipartite planar graph on $n$ vertices with parts $A, B$, and let $A^{\prime} \subset A$ be the set of vertices in $A$ with degree at least 3. Then the following are true.

1. The number of vertices in $A$ with degree exactly 2 is at least $e(A, B)-n-3|B|$;

2. $\left|A^{\prime}\right|<2|B|$;

3. $e\left(A^{\prime}, B\right)<6|B|$.

Proof. For each $i \geqslant 0$ let $A_{i}, A_{\leqslant i}$, and $A_{\geqslant i}$ denote the number of vertices in $A$ that have degree $i$ in $G$, degree at most $i$, and degree at least $i$, respectively. Because of planarity we have that $e\left(A^{\prime}, B\right)<2\left(\left|A^{\prime}\right|+|B|\right)$. Alternatively, $e\left(A^{\prime}, B\right) \geqslant 3\left|A^{\prime}\right|$ so it follows that $A_{\geqslant 3}=\left|A^{\prime}\right|<2|B|$, and so $e\left(A^{\prime}, B\right) \leqslant 2\left(\left|A^{\prime}\right|+|B|\right)<6|B|$, establishing the second and third items. Further, we can bound the number of edges between $A$ and $B$ as

$$
\begin{aligned}
e(A, B) & \leqslant A_{\leqslant 1}+2\left(|A|-A_{\leqslant 1}-A_{\geqslant 3}\right)+e\left(A^{\prime}, B\right) \\
& \leqslant A_{\leqslant 1}+2\left(|A|-A_{\leqslant 1}-\left|A^{\prime}\right|\right)+2\left(\left|A^{\prime}\right|+|B|\right) \\
& \leqslant 2|A|-A_{\leqslant 1}+2|B| .
\end{aligned}
$$

It follows that $A_{\leqslant 1} \leqslant 2|A|+2|B|-e(A, B)$. Finally, we see that $A_{2}=|A|-\left|A^{\prime}\right|-A_{\leqslant 1}>$ $e(A, B)-|A|-4|B|=e(A, B)-n-3|B|$, as required.

We are now in a position to prove our main theorem. First, let us give a rough sketch of the proof. Let $G$ be a path-pairable planar graph. We first partition the vertex set of $G$ into the set $A$ of vertices of small degree and the set $B$ of vertices of large degree. We can apply Lemma 7 to find that there are many edges in this cut. We shall then show that most vertices in $A$ have degree 2 in this bipartite graph. If $Y \subset A$ denotes the vertices of degree 2 , then we define a planar multigraph with vertex set $B$ where we join $x, y \in B$ whenever there is a $v \in Y$ joined to precisely $x$ and $y$. Now, using Corollary 6 , we are able to either find a vertex of linear degree in $B$, or we can find many pairs of multiedges in our multigraph that are far apart. This, however, allows us to find a pairing which contributes to more than $2 n$ edges between $A$ and $B$, a contradiction to planarity.

We restate Theorem 2 for convenience.

Theorem 2. There exists $c \geqslant 10^{-10^{10}}$ such that if $G$ is a path-pairable planar graph on $n$ vertices then $\Delta(G) \geqslant c n$.

Proof. Suppose $G$ is a path-pairable planar graph and fix some large constant $D$ so that $D^{-1} \leqslant 8.5 \cdot 10^{-6}$. Partition the vertex set of $G$ into sets $A$ and $B$, where $B=\{v \in V(G)$ : $d(v) \geqslant D\}$ and $A=V(G) \backslash B$. Since $e(G)<3 n$ it easily follows that $|B| \leqslant 6 D^{-1} n:=\varepsilon n$. Suppose that $\Delta(G)<c n$, where $c$ is sufficiently small (depending only on $D$ ) given by Lemma 7. More precisely, we may take $c=\frac{\varepsilon}{4 D^{2\lceil 4 / \varepsilon\rceil+1}}$. 
Our aim is to obtain a contradiction by showing such graph $G$ can not exist.

Of course, this is true if $c n<1$, so we shall assume throughout that $n \geqslant 1 / c$. By Lemma 7 (with $U=A$ ) we have that there are at least $2|A|-16 \varepsilon n \geqslant 2 n-18 \varepsilon n$ edges between $A$ and $B$.

Next, we shall show that there is a large subset of $A$ which induces a graph with maximum degree at most 2. To see this, let $A_{0}=A, B_{0}=B$. Suppose $A_{i}, B_{i}$ have been defined already. If there is a vertex $v \in A_{i}$ such that $d_{A_{i}}(v)>d_{B_{i}}(v)$, then let $A_{i+1}=A_{i} \backslash\{v\}$ and $B_{i+1}=B_{i} \cup\{v\}$. Notice that $e\left(A_{i+1}, B_{i+1}\right) \geqslant e\left(A_{i}, B_{i}\right)+1$, and so $e\left(A_{i+1}, B_{i+1}\right) \geqslant e(A, B)+i \geqslant 2 n-18 \varepsilon n+i$. Let $t \geqslant 0$ be such that there is no $v \in A_{t}$ with more neighbours in $A_{t}$ than in $B_{t}$. Observe that $t \leqslant 18 \varepsilon n$ (otherwise $e\left(A_{t}, B_{t}\right) \geqslant 2 n$ ), and accordingly $\left|B_{t}\right|=|B|+t \leqslant \varepsilon n+18 \varepsilon n=19 \varepsilon n$.

Let $X \subset A_{t}$ be the set of vertices in $A_{t}$ with at least 3 neighbours in $A_{t}$. Since every vertex in $A_{t}$ has more neighbours in $B_{t}$ than in $A_{t}$, we have that every vertex in $X$ has at least 3 neighbours in $B_{t}$. Therefore, by Lemma $8,|X| \leqslant 2\left|B_{t}\right|, e\left(X, B_{t}\right) \leqslant 6\left|B_{t}\right|$, and there are at least $e\left(A_{t}, B_{t}\right)-n-3\left|B_{t}\right| \geqslant e(A, B)-n-3\left|B_{t}\right|$ vertices in $A_{t}$ with exactly two neighbours in $B_{t}$. Let $A^{*}=A_{t} \backslash X$ and $B^{*}=B_{t} \cup X$. Now we have that every vertex in $A^{*}$ has at most 2 neighbours in $A^{*}$ and $\left|B^{*}\right| \leqslant 3\left|B_{t}\right| \leqslant 57 \varepsilon n$, so $\left|A^{*}\right| \geqslant n-57 \varepsilon n$. We have to make sure we still have many vertices in $A^{*}$ with exactly two neighbours in $B^{*}$. Notice that if a vertex $v \in A_{t}$ had two neighbours in $B_{t}$ and was not adjacent to any vertex in $X$ then $v \in A^{*}$ and $v$ still has exactly two neighbours in $B^{*}$. Therefore we only have to worry about the vertices in $A^{*}$ which are adjacent to some vertices in $X$. Observe that $e\left(X, A^{*}\right) \leqslant e\left(X, B_{t}\right) \leqslant 6\left|B_{t}\right|$, and so there are at least $e(A, B)-n-9\left|B_{t}\right| \geqslant(2 n-18 \varepsilon n)-n-9 \cdot 19 \varepsilon n=n-189 \varepsilon n$ vertices in $A^{*}$ with exactly 2 neighbours in $B^{*}$. Hence there are at most $189 \varepsilon n$ vertices in $A^{*}$ which do not have degree 2 in $B^{*}$.

In what follows, we shall deem certain edges of our graph as 'bad'. It will turn out that these edges are few in number, and, moreover, that paths connecting vertices in certain pairings, and which do not contain bad edges, must contribute to many edges between $A^{*}$ and $B^{*}$. Formally, we say that an edge $u v \in G$ is bad if one of the following holds:

1. (Type I) $u v \in G\left[B^{*}\right]$.

2. (Type II) $u v \in G\left[A^{*}\right]$ and $u$ (or $v$ ) has degree not equal to 2 in $B^{*}$.

3. (Type III) $u v \in G\left[A^{*}\right], d_{B^{*}}(u)=d_{B^{*}}(v)=2$, and $N_{B^{*}}(u) \neq N_{B^{*}}(v)$.

4. (Type IV) $u v \in G$, such that $u \in A^{*}, v \in B^{*}$, and $d_{B^{*}}(u) \geqslant 3$.

We have the following bound on the number of bad edges.

Claim 9. There are at most $1233 \varepsilon n$ bad edges.

Proof. We are going to bound the number of bad edges of each type.

Note that by planarity, there are at most $3\left|B^{*}\right|$ edges in $B^{*}$ so there are at most $3\left|B^{*}\right| \leqslant 171 \varepsilon n$ edges of Type I. 
Now, since every vertex in $A^{*}$ has at most two neighbours in $A^{*}$, each vertex in $A^{*}$ with degree not equal to 2 in $B^{*}$ contributes to at most two bad edges of Type II. As there are at most $189 \varepsilon n$ vertices in $A^{*}$ which do not have degree 2 in $B^{*}$, it follows that there are at most $378 \varepsilon n$ bad edges of Type II.

Let us consider bad edges of Type III. Since $G\left[A^{*}\right]$ has maximum degree 2, we can partition the edges of $G\left[A^{*}\right]$ into at most 3 matchings, $M_{1}, M_{2}, M_{3}$. It is well known (and easy to see) that contracting an edge in a planar graph preserves planarity. It follows that, for $i \in\{1,2,3\}$, we can contract the edges of $M_{i}$ while still preserving planarity. Denote this new graph by $\tilde{G}_{i}$ with vertex set $\tilde{A}_{i} \cup B^{*}$. Since $\tilde{G}_{i}$ is planar, from Lemma 8 we have that there are at most $2\left|B^{*}\right|$ vertices in $\tilde{G}_{i}$ with at least 3 neighbours in $B^{*}$. Therefore, at most $2\left|B^{*}\right|$ edges in $M_{i}$ can be bad of Type III. Hence, there are at most $6\left|B^{*}\right| \leqslant 342 \varepsilon n$ bad edges of Type III.

Finally, by Lemma 8 there can be at most $6\left|B^{*}\right| \leqslant 342 \varepsilon n$ bad edges of Type IV.

So in total there are at most $1233 \varepsilon n$ bad edges of any type.

Let $Y \subseteq A^{*}$ be the set of vertices with degree exactly 2 in $B^{*}$. We now define an auxiliary multigraph $G_{B^{*}}$ in the following way. The vertex set of $G_{B^{*}}$ is $B^{*}$ and for any two vertices $x, y \in B^{*}$, join $x$ to $y$ by an edge for every $v \in Y$ that is joined precisely to $x$ and $y$.

Claim 10. $G_{B^{*}}$ is planar.

Proof. This is clear since the bipartite graph $G\left[Y, B^{*}\right]$ between $Y$ and $B^{*}$ is planar, and contracting edges preserves planarity.

Apply Corollary 6 to the multigraph $G_{B^{*}}$ with $\varepsilon_{1}=\varepsilon_{2}=1 / 100$. Notice that if an edge in $G_{B^{*}}$ is incident with more than $\frac{1}{100}|Y|$ vertices then one of its endpoints has degree at least $\frac{1}{200}|Y|$. However, recall that we initially assumed $\Delta(G)<c n$, and certainly $c \leqslant 1 / 400$ by our choice of $D$. Accordingly, since $|Y| \geqslant n-189 \varepsilon n \geqslant n / 2$, we obtain a vertex of degree at least

$$
2 c|Y| \geqslant c n
$$

a contradiction.

So we may assume that there are at least $\frac{1}{100}\left(\begin{array}{c}|Y| \\ 2\end{array}\right)$ pairs of edges in $G_{B^{*}}$ which are at distance greater than 1 . Note that if $H$ is any graph on $n$ vertices with edge density at least $\delta$, then it is easy to greedily find a matching of size at least $\frac{\delta}{10} n$. It follows that we may select a collection of pairwise disjoint pairs $\mathcal{P}$ in $Y$, such that $|\mathcal{P}| \geqslant \frac{1}{1000}|Y| \geqslant \frac{1}{2000} n$, and such that for every $\{u, v\} \in \mathcal{P}$, their corresponding edges in $G_{B^{*}}$ are at distance greater than 1.

We need the following two claims.

Claim 11. Let $P$ be a path contained in $A^{*}$ which has at least two vertices and does not contain any bad edges. Then every vertex $v \in P$ has the same neighbourhood (of size 2 ) in $B^{*}$.

Proof. This is immediate from the definition of a bad edge. 
Claim 12. Let $u, v \in Y$ be two vertices whose corresponding edges in $G_{B^{*}}$ are at distance greater than 1 . Then any path in $G$ joining $u$ and $v$ either contains some bad edges, or uses at least 6 edges between $A^{*}$ and $B^{*}$.

Proof. Suppose $P$ is a path joining $u$ and $v$ which does not use any bad edges. By definition and using claim 11, all vertices of $V(P) \cap A^{*}$ are in $Y$, it can not have an edge inside $B^{*}$ and it must use 2 or 4 edges between $A^{*}$ and $B^{*}$. We may assume $P$ uses 4 edges as the other case follows from the same argument. Let $P=P_{1} e_{1} e_{2} P_{2} e_{3} e_{4} P_{3}$, where $\left\{e_{1}, e_{2}, e_{3}, e_{4}\right\}$ are edges between $A^{*}$ and $B^{*}$ and $P_{1}, P_{2}, P_{3}$ are paths inside $Y$. From claim 11 applied to $P_{1}, P_{2}$ and $P_{3}$ we deduce that the edge of $u$ in $G_{B^{*}}$ is at distance at most 1 to the edge of $v$ in $G_{B^{*}}$.

The proof of Theorem 2 is nearly complete. Indeed, since $G$ is path-pairable, there are edge-disjoint paths joining every pair of $\mathcal{P}$, and hence the pairs in $\mathcal{P}$ contribute to at least $6(|\mathcal{P}|-1233 \varepsilon n)$ edges between $A^{*}$ and $B^{*}$, by Claims 9 and 12 .

Let $P$ be the union of the vertices in $\mathcal{P}$ and let $U=A^{*} \backslash P$. Recall that $\left|A^{*}\right| \geqslant n-57 \varepsilon n$. Suppose first that $|U|<57 \varepsilon n$. It follows that

$$
2|\mathcal{P}|>(n-57 \varepsilon n)-57 \varepsilon n,
$$

so $|\mathcal{P}|>n / 2-57 \varepsilon n$. Then the above pairing contributes at least $6(n / 2-1290 \varepsilon n)=$ $3 n-7740 \varepsilon n$ edges between $A^{*}$ and $B^{*}$. But this is at least $2 n$ whenever $\varepsilon \leqslant 7740^{-1}$ which is guaranteed by our choice of $D$, a contradiction. Therefore, we may assume that $|U| \geqslant 57 \varepsilon n$. By Lemma 7 (since $c$ is small enough) there is a pairing of the vertices in $U$ which contributes to at least $2|U|-16 \cdot 57 \varepsilon n=2|U|-912 \varepsilon n$ edges between $A^{*}$ and $B^{*}$. Hence in total the number of edges between $A^{*}$ and $B^{*}$ is

$$
\begin{aligned}
& \geqslant 6(|\mathcal{P}|-1233 \varepsilon n)+2|U|-912 \varepsilon n \\
& =6(|\mathcal{P}|-1233 \varepsilon n)+2\left|A^{*}\right|-4|\mathcal{P}|-912 \varepsilon n .
\end{aligned}
$$

Recalling that $\left|A^{*}\right| \geqslant n-57 \varepsilon n$ and $|\mathcal{P}| \geqslant n / 2000$, we have that there are

$$
\begin{aligned}
& \geqslant 2|\mathcal{P}|+2(n-57 \varepsilon n)-6 \cdot 1233 \varepsilon n-912 \varepsilon n \\
& \geqslant 2 n+n / 1000-8424 \varepsilon n,
\end{aligned}
$$

edges between $A^{*}$ and $B^{*}$.

So by our choice of $D$ we get that $8424 \varepsilon \leqslant \frac{1}{1000}$, and so there are at least $2 n$ edges between $A^{*}$ and $B^{*}$, a contradiction to the planarity of $G$. It follows that there must exist a vertex of degree at least $c n$.

\section{Final Remarks and Open Problems}

It is worth observing that our proof relies only on the following three properties of a planar graph $G$ : contracting edges of $G$ preserves planarity, $G$ does not contain a $K_{5}$-minor, and any bipartite subgraph $H$ of $G$ has at most $2|H|$ edges. We remark that it is possible to 
generalize our result in the following sense. Given integers $t, c$, we say that a graph $G$ is $(t, c)$-good if $G$ is $K_{t}$-minor-free and any bipartite subgraph $H$ of $G$ has at most $2|H|+c$ edges. Moreover, define $\mathcal{G}_{t, c}$ to be the family of $(t, c)$-good graphs $G$ such that contracting edges of $G$ preserves $(t, c)$-goodness.

Theorem 13. For any integers $t, c$ there is a positive constant $C=C(t, c)$ such that the following holds. If $G$ is a path-pairable graph on $n$ vertices with $G \in \mathcal{G}_{t, c}$, then $\Delta(G) \geqslant C n$.

We have the following immediate corollary.

Corollary 14. For every non-negative integer $g$ there is a positive constant $C=C(g)$ such that the following holds. If $G$ is a path-pairable graph on $n$ vertices which has a 2-cell embedding on a surface with genus $g$, then $\Delta(G) \geqslant C n$.

Proof. We claim that $G \in \mathcal{G}_{3 g+5,2 g}$. Indeed, it follows from Euler's formula (see, e.g., [1]) that if $G$ is 2-cell embedded on a surface of genus $g$ then $n+m-f=2-g$, where $m$ is the number of edges $G$ and $f$ is the number of faces of the embedding. Since $2 m \geqslant 3 f$ $(2 m \geqslant 4 f$ if $G$ is triangle-free) it follows that $e(G) \leqslant 3 n+3 g-6(e(G) \leqslant 2 n+2 g-4$ if $G$ is triangle-free). In particular, if $G$ is bipartite then $e(G) \leqslant 2 n+2 g-4 \leqslant 2 n+2 g$. Suppose for contradiction that $G$ contains a $K_{3 g+5}$-minor. Then $K_{3 g+5}$ could be 2 -cell embedded on a surface of genus $g$, hence $\left(\begin{array}{c}3 g+5 \\ 2\end{array}\right)=e\left(K_{3 g+5}\right) \leqslant 12 g+9$, which is easily seen to be a contradiction.

Sketch of a proof of Theorem 13. The proof is essentially the same as the proof of Theorem 2. Certain changes have to be made in the preparatory lemmas first.

Corollary 6 generalizes trivially to multigraphs with no $K_{t}$-minors.

In Lemma 7 we only use the fact that any subgraph $H$ of a planar graph has at most $3|H|$ edges. Observe that if $G \in \mathcal{G}_{t, c}$ then any subgraph of $H$ of $G$ has at most $4|H|+2 c$ edges. One can therefore modify the proof, at the expense of a worse constant in front of $\varepsilon n$ in the conclusion of the Lemma.

In the proof of Lemma 8 we only use the fact that a bipartite subgraph $H$ of a planar graph does not use more than $2|H|$ edges. The lemma can be therefore modified to work for graphs in $\mathcal{G}_{t, c}$ by introducing some additive constants, depending only on $c$, to the inequalities in every part of the Lemma.

In the proof of Theorem 2 all the estimates remain correct by taking $\varepsilon$ small enough.

Note that we also need that $\mathcal{G}_{t, c}$ is closed under edge contractions in order to estimate the number of "bad edges", as in Claim 9 (there we used that contracting edges preserves planarity).

We believe that the condition on the number of edges in bipartite subgraphs can be omitted while still ensuring the existence of a vertex of linear degree. We therefore make the following conjecture.

Conjecture 15. For any $t$ there exists a constant $c=c(t)$ such that every path-pairable graph on $n$ vertices without a $K_{t}$ minor must contain a vertex of degree at least $c n$. 
Finally, recall that we defined $\Delta_{\min }^{p}(n)$ to be the minimum of $\Delta(G)$ over all $n$-vertex path-pairable planar graphs $G$. We have shown that $\Delta_{\text {min }}^{p}(n)$ grows linearly in $n$; however, as mentioned in the Introduction, the constants in the upper and lower bounds are quite far apart. We close with the following problem.

Problem 16. Determine $\Delta_{\min }^{p}(n)$ for sufficiently large $n$.

We do not know if our construction yielding the upper bound of $2 n / 3$ is optimal, and a significant improvement on our lower bound would be very interesting.

\section{Aknowledgments}

We would like to thank David Wood for his helpful suggestion that our theorem would also hold for bounded genus graphs following the same proof. We would also like to express our thanks to the anonymous referees for many helpful suggestions.

\section{References}

[1] BollobÁs, B. Modern Graph Theory, vol. 184 of Graduate Texts in Mathematics. Springer-Verlag, New York, 1998.

[2] Bollobás, B., And Thomason, A. Highly linked graphs. Combinatorica 16, 3 (1996), 313-320.

[3] Csaba, L., Faudree, R., Gyárfás, A., Lehel, J., And Schelp, R. Networks communicating for each pairing of terminals. Networks 22 (1992), 615-626.

[4] Faudree, R., Gyárfás, A., And Lehel, J. Minimal path pairable graphs. Congressus Numerantium 88 (1992), 111-128.

[5] Faudree, R. J. Properties in path-pairable graphs. New Zealand Journal of Mathematics 21 (1992), 91-106.

[6] Faudree, R. J., GyÁrfás, A., And Lehel, J. Path-pairable graphs. Journal of Combinatorial Mathematics and Combinatorial Computing 20 (1999), 145-157.

[7] Frank, A. Packing paths, circuits, and cuts - a survey. In Paths, Flows and VLSILayouts, B. Korte, L. Lovász, and A. S. H-J. Prömel, Eds. Springer Verlag, 1990, pp. $47-100$.

[8] Frieze, A. M. Disjoint Paths in Expander Graphs via Random Walks: a Short Survey. Springer Berlin Heidelberg, Berlin, Heidelberg, 1998, pp. 1-14.

[9] Győri, E., Mezei, T. R., And MÉszÁros, G. Terminal pairability in complete graphs. Journal of Combinatorial Mathematics and Combinatorial Computing, submitted, arXiv:1605.05857.

[10] Győri, E., Mezei, T. R., And MÉszÁros, G. Note on terminal-pairability in complete grid graphs. Discrete Mathematics 340 (2017), 988-990.

[11] Hirata, T., Kubota, K., and Saito, O. A sufficient condition for a graph to be weakly k-linked. Journal of Combinatorial Theory, Series B 36, 1 (1984), 85-94. 
[12] HuCK, A. A sufficient condition for graphs to be weaklyk-linked. Graphs and Combinatorics 7, 4 (Dec 1991), 323-351.

[13] Kawarabayashi, K., Kobayashi, Y., and Reed, B. The disjoint paths problem in quadratic time. Journal of Combinatorial Theory, Series B 102, 2 (2012), 424-435.

[14] Kubicka, E., Kubicki, G., And Lehel, J. Path-pairable property for complete grids. Combinatorics, Graph Theory, and Algorithms 1 (1999), 577-586.

[15] Lipton, R., And TaRjan, R. A separator theorem for planar graph. SIAM Journal on Applied Mathematics 36 (1979), 177-189.

[16] MÉszÁros, G. On path-pairability in the cartesian product of graphs. Discussiones Mathematicae Graph Theory 36 (2016), 743-758.

[17] Middendorf, M., And Pfeiffer, F. On the complexity of the disjoint paths problem. Combinatorica 13, 1 (1993), 97-107.

[18] Robertson, N., And Seymour, P. Graph minors. XIII. The disjoint paths problem. Journal of Combinatorial Theory, Series B 63 (1995), 65-110.

[19] Thomas, R., and Wollan, P. An improved linear edge bound for graph linkages. European Journal of Combinatorics 26, 3 (2005), 309 - 324. Topological Graph Theory and Graph Minors, second issue.

[20] Vygen, J. Disjoint paths. Report No. 94816-OR, Research Institute for Discrete Mathematics, University of Bonn (1994). 